\title{
INTERPOLATION OF OPERATORS WITH CHANGE OF MEASURES
}

BY

\author{
E. M. STEIN AND G. WEISS
}

Introduction. This paper has two purposes. First, it is to give general interpolation theorems which allow one to change measures simultaneously with changing exponents of the $L^{p}$ classes concerned. These theorems are generalizations of theorems of M. Riesz [7] and Marcinkiewicz [4]. The idea of interpolating between measure seems to have been implicit for some time. The general situation has, however, only recently been investigated. (See e.g. [2] and [8].)

Our second purpose is to apply these techniques. In $\S \S 3$ and 4 , we shall prove theorems for unbounded orthonormal systems which generalize known results for bounded orthonormal systems. These theorems are extensions of theorems of Paley, Marcinkiewicz and Zygmund [5], and Pitt [6].

$\$ 1$ will be devoted to necessary definitions and statements of needed known results. $\$ 2$ will contain the general interpolation theorems.

1. Definitions and statement of general results. Let $(M, \mathfrak{M}, \mu)$ and $(N, \mathfrak{R}, \nu)$ be two measure spaces. We will consider only measurable, real or complex-valued functions on these two spaces. In this paper we will establish a generalization of the well known convexity theorem of M. Riesz (Theorem (1.1) below) and give some applications of this new theorem to certain orthonormal series. In order to do this we will need to establish some notation and give a few definitions.

Suppose $T$ is a mapping of a class of functions on $M$ into a class of functions on $N . T$ is called a sublinear operator if it satisfies the following properties:

(i) If $f=f_{1}+f_{2}$ and $T f_{i}(i=1,2)$ are defined then $T f$ is defined;

(ii) $\left|T\left(f_{1}+f_{2}\right)\right| \leqq\left|T f_{1}\right|+\left|T f_{2}\right|$ almost everywhere;

(iii) For any scalar $k$, we have $|T(k f)|=|k||T f|$ almost everywhere.

Let $p, q \geqq 1$ be two real numbers. We say that $T$ is of type $(p, q)$ in case $T$ is defined for all functions $f$ in $L^{p}(M, \mathfrak{M}, \mu)$ and there exists a positive number, $K$, independent of $f$ such that

$$
\|T f\|_{q, \nu} \leqq K\|f\|_{p, \mu}
$$

where

Received by the editors September 12, 1956. 


$$
\|T f\|_{q, \nu}=\left(\int_{N}|T|^{q} d \nu\right)^{1 / q}
$$

and

$$
\|f\|_{p, \mu}=\left(\int_{M}|f|^{p} d \mu\right)^{1 / p} .
$$

We can also introduce types $(\infty, q),(p, \infty)$ and $(\infty, \infty)$ by allowing the norms in the first inequality to be $L^{\infty}$-norms; i.e. $\|g\|_{\infty}=$ essential supremum of $|g|$.

If $h$ is a function defined on $N$ and $y>0$, we will let $E_{y} \in \mathfrak{R}$ be the set of all $x$ in $N$ such that $|h(x)|>y$. The distribution function, $\lambda=\lambda_{h}$, of $|h|$ will then be defined by letting

$$
\lambda(y)=\nu\left(E_{y}\right) \quad \text { for all } y>0 .
$$

Now suppose $T$ is of type $(p, q)$ and set $h=T f$ for $f$ in $L^{p}(M, \mathfrak{M}, \mu)$. Letting $\lambda$ be the distribution function of $|h|$ we see that

$$
y(\lambda(y))^{1 / q}=\left(\int_{E_{\nu}} y^{q} d \nu\right)^{1 / q} \leqq\left(\int_{E_{\nu}}|h|^{q} d \nu\right)^{1 / q} \leqq\left(\int_{N}|h|^{q} d \nu\right)^{1 / q} \leqq K\|f\|_{p, \mu} .
$$

Thus we have shown that

$$
\lambda(y) \leqq\left(K y^{-1}\|f\|_{p, \mu}\right)^{q} .
$$

We shall say that a sublinear operator, $T$, is of weak type $(p, q)$ in case the domain of $T$ includes $L^{p}(M, \mathfrak{M}, \mu)$ and the above inequality is satisfied for all $f$ in this space, with $K$ independent of $f$. If $q=\infty$ we let weak type be the same as type $(p, \infty)$.

One form of the convexity theorem of M. Riesz is the following:

TheOREM (1.1). If $T$ is a sublinear operator of types $\left(p_{0}, q_{0}\right)$ and $\left(p_{1}, q_{1}\right)$, with constants $K_{0}$ and $K_{1}$ respectively, and if $p=p_{t}, q=q_{t}$, where $0 \leqq t \leqq 1$, are defined by

$$
1 / p=(1-t)\left(1 / p_{0}\right)+t\left(1 / p_{1}\right) \text { and } 1 / q=(1-t)\left(1 / q_{0}\right)+t\left(1 / q_{1}\right)
$$

then $T$ is of type $(p, q)$. More precisely, for any $f$ in $L^{p}(M, \mathfrak{M}, \mu) T f$ is defined and

$$
\|T f\|_{q, \nu} \leqq K_{0}^{(1-t)} K_{1}^{t}\|f\|_{p, \mu} .
$$

Inequality (1.2) asserts that the norm of the operator $T$, as a function of $t$, is logarithmically convex. M. Riesz [7] proved this result in a somewhat different setting, obtaining inequalities involving certain linear forms. His theorem, however, is essentially the same as (1.1) under the added hypotheses that $T$ is a linear operator acting on real-valued functions and $p_{j} \leqq q_{j}(j=0,1)$. 
The fact that Theorem (1.1) is true for a linear operator, $T$, acting on complex-valued functions is due to Thorin [9]. The extension to sublinear operators is due to Calderón and Zygmund [1].

There are sublinear operators, however, that fail to be one or both of the types $\left(p_{i}, q_{i}\right)(i=0,1)$, but are of weak types $\left(p_{i}, q_{i}\right)$, and, in addition, are of types $\left(p_{t}, q_{t}\right)$ when $0<t<1$. An example is furnished by mapping a Lebesgue integrable function defined on $(0,2 \pi)$ into its conjugate function. Kolmogoroff showed [3] that this linear operator is of weak type $(1,1)$ and it is easy to check that it is also of type $(2,2)$. A well known inequality of M. Riesz [10] asserts, in particular, that this mapping is of type $(p, p)$ for all $p$ satisfying $1<p \leqq 2$. An important sublinear, but not linear, operator with exactly these properties is the mapping of a function in $L^{1}(0,2 \pi)$ into its Hardy-Littlewood maximal function [10].

Marcinkiewicz showed [4] that the above facts are special cases of a more general situation. His theorem can be stated as follows $\left({ }^{1}\right)$ :

ThEOREM (1.3). If $T$ is a sublinear operator of weak types $\left(p_{i}, q_{i}\right)$, where $p_{i} \leqq q_{i}, i=0,1$, then $T$ is of type $\left(p_{t}, q_{t}\right)$ whenever $0<t<1$.

We shall now show that both Theorems (1.1) and (1.3) can be put in more general form.

2. Generalizations of the theorems of Riesz and Marcinkiewicz. Suppose $\mu_{0}$ and $\mu_{1}$ are two measures on $\mathfrak{M}$. Let $\mu=\mu_{0}+\mu_{1}$, then $\mu_{0}$ and $\mu_{1}$ are each absolutely continuous with respect to $\mu$. Thus, by the Radon-Nikodym theorem, there exists two functions, $\alpha_{0}$ and $\alpha_{1}$, such that for any set $E$ in $\mathfrak{M}$

$$
\mu_{j}(E)=\int_{E} \alpha_{j} d \mu \quad(j=0,1) .
$$

In the following we will assume that $\alpha_{0}$ and $\alpha_{1}$ are never zero. This is equivalent to asserting that the sets of measure zero with respect to $\mu_{j}$ $(j=0,1)$ are the same as the sets of measure zero with respect to $\mu$. Thus, in the various measure spaces that we will consider, the equivalence classes of functions will always be the same (where, as is well known, two functions are called equivalent if and only if they differ only on a set of measure 0 ).

If $0 \leqq s \leqq 1$ we define the measure $\mu_{s}$ on $\mathfrak{M}$ by

$$
\mu_{s}(E)=\int_{E} \alpha_{0}^{(1-s)} \alpha_{1}^{s} d \mu
$$

for each $E$ in $\mathfrak{M}$.

The following lemma gives us a certain uniqueness result for $\mu_{s}$.

(1) Actually, Marcinkiewicz announced this result for $p_{i}=q_{i}$. The above theorem and its proof is given in [11]. The theorem is true for more general operators than sub-linear ones. We refer the reader to [11] for further details. 
Lemma (2.2). Suppose $\mu_{j}$ has the two representations

$$
\int_{E} \alpha_{j} d \mu=\mu_{j}(E)=\int_{E} \bar{\alpha}_{j} d \bar{\mu} \quad(j=0.1) .
$$

Then

$$
\int_{E} \alpha_{0}^{(1-s)} \alpha_{1}^{s} d \mu=\int_{E} \bar{\alpha}_{0}^{(1-s)} \bar{\alpha}_{1}^{s} d \bar{\mu}
$$

for each $E$ in $\mathfrak{M}$ and $0 \leqq s \leqq 1$.

Proof. From (2.3) we have, for any $\mu_{j}$-integrable $f$,

$$
\int_{E} f \alpha_{j} d \mu=\int_{E} f \bar{\alpha}_{j} d \bar{\mu} \quad(j=0,1 \text { and all } E \text { in } \mathfrak{M}) .
$$

In particular, putting $f=1 / \alpha_{j}$, we obtain

$$
\mu(E)=\int_{E} d \mu=\int_{E}\left(\bar{\alpha}_{j} / \alpha_{j}\right) d \bar{\mu}, \quad(j=0,1) .
$$

Thus $\bar{\alpha}_{0} / \alpha_{0}=\bar{\alpha}_{1} / \alpha_{1}$ almost everywhere. Or, equivalently, $\alpha_{0} / \alpha_{1}=\bar{\alpha}_{0} / \bar{\alpha}_{1}$ almost everywhere.

Using this last fact and (2.4) with $f=\left(\alpha_{1} / \alpha_{0}\right)^{s}$ we have:

$$
\begin{aligned}
\int_{E} \alpha_{0}^{(1-s)} \alpha_{1}^{s} d \mu & =\int_{E}\left(\alpha_{0} / \alpha_{1}\right)^{s} \alpha_{0} d \mu=\int_{E}\left(\alpha_{1} / \alpha_{0}\right)^{s} \bar{\alpha}_{0} d \bar{\mu}=\int_{E}\left(\bar{\alpha}_{1} / \bar{\alpha}_{0}\right)^{s} \bar{\alpha}_{0} d \bar{\mu} \\
& =\int_{E} \bar{\alpha}_{0}^{(1-s)} \bar{\alpha}_{1}^{s} d \bar{\mu}
\end{aligned}
$$

which is the desired conclusion.

We now develop some notation needed for the statement of the next lemma, which contains the basic idea in the proof of the main theorem.

Given two distinct positive real numbers, $p_{0}$ and $p_{1}$, let $\zeta$ be the measure on $\mathfrak{M}$ defined by

$$
\zeta(E)=\int_{E}\left(\alpha_{0}^{p_{1}} \alpha_{1}^{-p_{0}}\right)^{1 /\left(p_{1}-p_{0}\right)} d \mu .
$$

Let $s=s(t)$ be the number $s=\left(t p_{t}\right) / p_{1}$, where $0 \leqq t \leqq 1$. Then $1-s=(1-t) p_{t} / p_{0}$, since $(1-t) p_{t} / p_{0}+t p_{t} / p_{1}=1$.

Lemma (2.6). There exists a linear mapping, $A$, of the space of all measurable functions on $M$ into itself such that its restriction to any of the spaces $L^{p_{t}}\left(M, \mathfrak{M}, \mu_{s(t)}\right)$, where $0 \leqq t \leqq 1$, is an isometry onto the space $L^{m}(M, M i, \zeta)$.

Proof. We define $A$ by letting $A f=\left(\alpha_{0} / \alpha_{1}\right)^{1 /\left(p_{0}-p_{1}\right)} f$. Then we have: 


$$
\int_{M}|A f|^{p_{t}} d \zeta=\int_{M}\left(\alpha_{0} / \alpha_{1}\right)^{\left(p_{t} /\left(p_{0}-p_{1}\right)\right.}|f|^{p_{t}}\left(\alpha^{p_{1}} \alpha_{1}^{-p_{0}}\right)^{1 /\left(p_{1}-p_{0}\right)} d \mu .
$$

But using the fact $p_{t}=p_{0} p_{1} /\left(p_{1}(1-t)+p_{0} t\right)$, we obtain:

$$
p_{0}\left(p_{t}-p_{1}\right) / p_{0}-p_{1}=p_{0} p_{t}-\left[p_{t} p_{1}(1-t)+p_{t} p_{0} t\right] /\left(p_{0}-p_{1}\right)=(1-t) p_{t} .
$$

Thus $\left(p_{t}-p_{1}\right) /\left(p_{0}-p_{1}\right)=(1-t) p_{t} / p_{0}=1-s(t)=$ the exponent of $\alpha_{0}$. Similarly, $\left(p_{t}-p_{0}\right) /\left(p_{1}-p_{0}\right)=t p_{t} / p_{1}=s(t)$. Thus we have shown that

$$
\int_{M}|A f|^{p_{t}} d \zeta=\int_{M}|f|^{p_{t}} d \mu_{s(t)}
$$

But this asserts that $A$ is an isometry. That $A$ is onto is clear, since it has an inverse. This completes the proof of the lemma.

Now suppose that we are given two measures, $\nu_{0}$ and $\nu_{1}$, on the $\sigma$-ring of measurable sets $\mathfrak{R}$. Then for any $r$ satisfying $0 \leqq r \leqq 1$, we can define the measure $\nu_{r}$ on $\mathfrak{R}$. Let $\beta_{0}$ and $\beta_{1}$ be the functions giving us the measures $\nu_{0}$ and $\nu_{1}$, respectively, in a manner completely analogous to the way $\alpha_{0}$ and $\alpha_{1}$ gave us the measures $\mu_{0}$ and $\mu_{1}$. Here, again, we assume that neither $\beta_{0}$ nor $\beta_{1}$ are ever 0 . If two positive real numbers, $q_{0}$ and $q_{1}$, are given, we define the measure $\xi$ on $\mathfrak{N}$ by letting

$$
\xi(F)=\int_{F}\left(\beta_{0}^{q_{1}} \beta_{1}^{-q_{0}}\right)^{1 /\left(q_{1}-q_{0}\right)} d \nu
$$

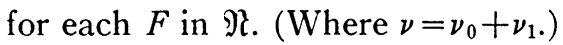

We shall let $r(t)=t q_{t} / q_{1}$. Then $1-r(t)=(1-t) q_{t} / q_{0}$. Applying Lemma (2.6) to this situation we obtain a linear mapping, $B$, of the space of all measurable functions on $N$ into itself, which, when restricted to the spaces $L^{q_{t}}\left(N, \mathfrak{R}, \nu_{r(t)}\right)$ is an isometry onto $L^{q_{t}}(N, \mathfrak{R}, \xi)$.

The main result in this section is the following theorem.

THEOREM (2.9). Let $T$ be a sublinear operator, mapping functions defined on $M$ into functions defined on $N$, having the following two properties:

(i) The domain of $T$ includes $L^{p_{0}}\left(M, \mathfrak{M}, \mu_{0}\right) \cup L^{p_{1}}\left(M, \mathfrak{M}, \mu_{1}\right)$.

(ii) If $f$ is in $L^{p_{i}}\left(M, \mathfrak{M}, \mu_{j}\right)(j=0,1)$ let

$$
F_{y}=\{x \text { in } N ;|k(x) h(x)|>y\},
$$

where $h=T f, k=\left(\beta_{0}^{\prime} \beta_{1}\right)^{1 /\left(q_{0}-q_{1}\right)}$ and $y>0$. Then, letting $\lambda(y)=\xi\left(F_{y}\right)$ we have

$$
\lambda(y) \leqq\left(\left(K_{j} / y\right)\left\{\|f\|_{p_{j}, \mu_{j}}\right\}\right)^{q_{i}},
$$

where $K_{j}$ is a positive number, independent of $f$ in $L^{p_{i}}\left(M, \mathfrak{M}, \mu_{j}\right)$, and $p_{j} \leqq q_{j}$.

Then $T$ is defined on $L^{p_{t}}\left(M, \mathfrak{M}, \mu_{s(t)}\right)$, for $0<t<1$, and if $f$ is in this space

$$
\|T f\|_{q_{t}, \nu_{r(t)}} \leqq K_{t}\|f\|_{p_{t}, \mu_{s(t)}}
$$

where $K_{t}$ is independent of $f$. 
We observe that if each of the functions $\alpha_{0}, \alpha_{1}, \beta_{0}, \beta_{1}$ is identically $1,(2.9)$ reduced to Theorem (1.3).

Proof of Theorem (2.9). We first show that the domain of $T$ includes $L^{p_{t}}\left(M, \mathfrak{M}, \mu_{s(t)}\right)$. If $f$ is in this last space, then $A f$ is in $L^{p_{t}}(M, \mathfrak{M}, \zeta)$, by Lemma (2.6). Let $A f=g_{0}+g_{1}$, where, for $x$ in $M$

$$
g_{0}(x)=\left\{\begin{array}{cc}
{[A f](x)} & \text { if }|[A f](x)| \leqq 1 \\
0 & \text { if }|[A f](x)|>1
\end{array}\right.
$$

$g_{1}$ is then defined by $g_{1}=A f-g_{0}$.

Assume that $p_{0}>p_{1}$ (if $p_{0}<p_{1}$ the argument is exactly the same with the roles of $p_{0}$ and $p_{1}$ interchanged). Then, clearly

$$
\left|g_{0}\right|^{p_{0}} \leqq|A f|^{p_{t}}, \quad\left|g_{1}\right|^{p_{1}} \leqq|A f|^{p_{t}} .
$$

Since $|A f|^{p_{t}}$ is integrable with respect to $\zeta$ we have that $g_{0}$ is in $L^{p_{0}}(M, \mathfrak{M}, \zeta)$ and $g_{1}$ is in $L^{p_{1}}(M, \mathfrak{M}, \zeta)$. Thus $A^{-1} g_{0}$ is in $L^{p_{0}}\left(M, \mathfrak{M}, \mu_{0}\right)$ and $A^{-1} g_{1}$ is in $L^{p_{1}}\left(M, \mathfrak{M}, \mu_{1}\right)$. Hence, both $T\left(A^{-1} g_{0}\right)$ and $T\left(A^{-1} g_{1}\right)$ are defined. But $A^{-1} g_{0}$ $+A^{-1} g_{1}=f$ and, thus, by the first property in the definition of sublinear operators, $T f$ is defined.

Now let us consider the operator $S=B T A^{-1}$ that maps $L^{p_{t}}(M, \mathfrak{M}, \zeta)$ into $L^{q t}(N, \mathfrak{R}, \xi)$. The above argument shows that $S$ is well defined and, furthermore, it is easy to check that $S$ is sublinear. Since, by Lemma (2.6) $\left\|A^{-1}\right\|_{p_{j}, \mu_{j}}$ $=\|f\|_{p_{j}, \zeta}(j=0,1)$, inequality $(2.10)$ asserts precisely that $S$ is of weak types $\left(p_{0}, q_{0}\right)$ and $\left(p_{1}, q_{1}\right)$ when we have the measures $\zeta$ on $\mathfrak{M}$ and $\xi$ on $\mathfrak{N}$. Thus by (1.3) there exists a constant, $K_{t}$, independent of $g$ in $L^{p_{t}}(M, \mathfrak{M}, \zeta)$, such that

$$
\|S g\|_{q_{t}, \xi} \leqq K_{t}\|g\|_{p_{t}, \zeta} .
$$

But, by the isometric properties of $A$ and $B$, this is equivalent to the assertion that

$$
\|T f\|_{q_{t}, r_{r(t)}} \leqq K_{t}\|f\|_{p_{t}, \mu_{s(t)}}
$$

for all $f$ in $L^{p_{t}}\left(M, \mathfrak{M}, \mu_{s(t)}\right)$. This proves the theorem.

The above proof also yields a more general formulation of Theorem (1.1) as well. More precisely, we have:

THEOREM (2.11). If $T$ is a sublinear operator statisfying

$$
\|T f\|_{a_{j}, \nu_{j}} \leqq K_{j}\|f\|_{p_{j}, \mu_{j}}
$$

for all $f$ in $L^{p_{i}}\left(M, \mathfrak{M}, \mu_{j}\right), j=0,1 ;$ then, for $0 \leqq t \leqq 1$,

$$
\|T f\|_{q_{t}, \nu_{r(t)}} \leqq K_{0}^{(1-t)} K_{1}^{t}\|f\|_{p_{t}, \mu_{s(t)}}
$$

for all $f$ in $L^{p_{t}}\left(M, \mathfrak{M}, \mu_{s(t)}\right)$, where $p_{0} \neq p_{1}$ and $q_{0} \neq q_{1}$ are real numbers not exceeded by 1 .

This theorem was proved by a different method by one of us [8] in case 
$T$ is linear. In that proof, the assumption that $p_{0} \neq p_{1}$ and $q_{0} \neq q_{1}$ is not needed. In fact, this assumption can also be done away with in (2.11) by combining the methods in [1] and [8].

REMARKs. (1). It is sometimes useful in the applications of Theorems (2.9) and (2.10) to rewrite the inequalities obtained in the following way. Let $a_{j}=\alpha_{j}^{1 / p_{j}}$ and $b_{j}=\alpha_{j}^{1 / q_{i}}$, for $j=0,1$. Then

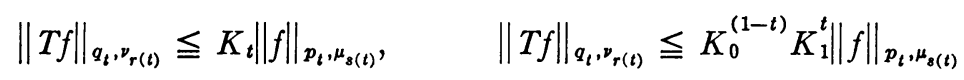

become

$$
\begin{aligned}
& \left\|(T f) b_{0}^{(1-t)} b_{1}^{t}\right\|_{q_{t}, \nu} \leqq K_{t}\left\|f a_{0}^{(1-t)} a_{1}^{t}\right\|_{p_{t}, \mu} \\
& \left\|(T f) b_{0}^{(1-t)} b_{1}^{t}\right\|_{q_{t}, \nu} \leqq K_{0}^{(1-t)} K_{1}^{t}\left\|f a_{0}^{(1-t)} a_{1}^{t}\right\|_{p_{t}, \mu}
\end{aligned}
$$

respectively.

(2). The restriction that neither $\alpha_{0}$ nor $\alpha_{1}$ are ever zero seems unavoidable. This is so since the $L^{p}$ spaces we consider are spaces of equivalence classes of functions and we need the fact that these equivalence classes remain invariant under the changes of measures we dealt with. In fact, Theorems (2.9) and (2.11) were originally proved using the measures $d \mu_{0}=\alpha_{0} d \mu$ and $d \mu_{1}=\alpha_{1} d \mu$; that is, the functions $\alpha_{0}$ and $\alpha_{1}$ were given at the outset. Dr. L. Hörmander suggested to us that we define $d \mu=d \mu_{0}+d \mu_{1}$, thereby allowing treatment of the most general case.

3. Theorems of Marcinkiewicz and Zygmund. As a first application of (2.9) we will establish certain generalizations of Paley's theorems on orthonormal series. These generalizations are the same as those of Marcinkiewicz and Zygmund [5], though the proofs given here are quite different. We shall need these theorems in the next section $\left({ }^{2}\right)$.

Let $\left\{\phi_{n}\right\}, n=1,2, \cdots$, be an orthonormal system of functions defined on the measure space $(M, \mathfrak{M}, \mu)$. We will assume that for a certain $k>2$

$$
\left(\int\left|\phi_{n}\right|^{k} d \mu\right)^{1 / k} \leqq M_{n}<\infty
$$

for each positive integer $n$. We also include the case $k=\infty$ by assuming that, in this case, $\left\|\phi_{n}\right\|_{\infty}=M_{n}$. All the following assertions hold for $k=\infty$, the arguments being valid after only slight changes. We will also denote by $l$ the number satisfying $1 / k+1 / l=1$. We will also assume that $\left\{\phi_{n}\right\}$ is complete, though quite a few of the following results go through without this assumption.

Given a function, $f$, in $L^{p}(M, \mathfrak{M}, \mu), l \leqq p \leqq 2$, then, for each $n, \int \phi_{n} f d \mu=c_{n}$ is a finite number. This is so because (3.1) asserts that $\phi_{n}$ is in $L^{k}(M, \mathfrak{M}, \mu)$ and this, together with the fact that $\phi_{n}$ is in $L^{2}(M, \mathfrak{M}, \mu)$, implies that $\phi_{n}$ is

(2) In [11] A. Zygmund proves the original Paley theorems by means of the Marcinkiewicz interpolation theorem, which method is here adopted for the more general situation. 
in $L^{q}(M, \mathfrak{M}, \mu)$ where $1 / p+1 / q=1$, since we must have $2 \leqq q \leqq k$. Thus the mapping carrying $f$ into the sequence $\left\{c_{n}\right\}$ is a well defined transformation.

We have, then, the following theorem which, in the case $k=\infty$ and $M_{1}=M_{2}=\cdots=M_{n}=\cdots$, reduces to the original theorem of Paley.

Theorem (3.2). If $l<p \leqq 2$ then

$$
\left(\sum_{n=1}^{\infty}\left|c_{n}\right|^{p} D_{n}^{p-2}\right)^{1 / p} \leqq K_{p, k}\|f\|_{p, \mu}
$$

where $D_{n}=\left(M_{n}^{*}\right)^{k /(k-2)} n^{(k-1) /(k-2)}, M_{n}^{*}=\max _{j \leqq n} M_{j}$.

Proof. Letting the operator $T$ be the map $f \rightarrow\left\{c_{n}\right\}$, we can apply (2.9) in the following way. Let $(N, \mathfrak{N}, \nu)$ be the positive integers with measure 1 assigned to each point, and using the notation established in (2.12), set $b_{0}(n)$ $=\left(M_{n}^{*}\right)^{-1} \cdot n^{-1 / l}, b_{1}(n)=1$, for each $n$ in $N, a_{0}(x) \equiv 1 \equiv a_{1}(x)$, for $x$ in $M$, $p_{0}=l=q_{0}$ and $p_{1}=2=q_{1}$. The measure $\xi$ then, evaluated at each $n$ in $N$, is easily seen to be

$$
\xi(n)=\left(M_{n}^{*}\right)^{2 k /(2-k)} n^{2(k-1) /(2-k)} .
$$

If we can then show inequality (2.10), the conclusion of (2.9), for this case, is easily seen to be (3.3) (letting $t$ be that number satisfying $1 / p$ $\left.=(1-t) / p_{0}+t / p_{1}=(1-t) / l+t / 2\right)$. But if $p=q=2$ (i.e. $\left.t=1\right)$ (3.3) is just Bessel's inequality. Hence we clearly have (2.10) for this case (see argument in $\S 1$ that shows that type $(p, q)$ implies weak type $(p, q))$. The case $p=q=l$ reduces to showing that $\lambda(y)$, the distribution function of

$$
\left|\left[b_{0}(n)\right]^{l /(l-2)} c_{n}\right|=\left\{\left(M_{n}^{*}\right)^{l /(-l+2)} n^{1 /(2-l)}\left|c_{n}\right|\right\}=\left\{\left(M_{n}^{*}\right)^{k /(k-2)} n^{(k-1) /(k-2)}\left|c_{n}\right|\right\}
$$

with respect to the measure $\xi$, satisfies

$$
\lambda(y) \leqq\left(K_{0} y^{-1}\|f\|_{l, \mu}\right)^{l}
$$

for all $f$ in $L^{l}(M, \mathfrak{M}, \mu)$, and $y>0$.

Let $E_{y}=\left\{n ;\left(M_{n}^{*}\right)^{k /(k-2)} n^{(k-1) /(k-2)}\left|c_{n}\right|>y\right\}$, then $\lambda(y)=\xi\left(E_{y}\right)$. On the other hand,

$$
\left|c_{n}\right|=\left|\int \phi_{n} f d \mu\right| \leqq M_{n}^{*}\|f\|_{l, \mu}
$$

by Hölder's inequality and (3.1). Thus if

$$
F_{y}=\left\{n:\left[\left(M_{n}^{*}\right)^{k /(k-2)} n^{(k-1) /(k-2)}\right] M_{n}^{*}\|f\|_{l, \mu}>y\right\}
$$

we have $E_{y} \subset F_{y}$ and, consequently, $\lambda(y) \leqq \xi\left(F_{y}\right)$. Thus, it suffices to show that

$$
\xi\left(F_{y}\right) \leqq\left(K_{0} y^{-1}\|f\|_{l, \mu}\right)^{l} .
$$

Let $n_{0}$ be the first integer in $F_{y}$. We then have, since $M_{n_{0}}^{*} \leqq M_{n}^{*}$ if $n_{0} \leqq n$, 


$$
\begin{aligned}
\xi\left(F_{y}\right) & =\sum_{n \in F_{y}}\left(M_{n}^{*}\right)^{2 k /(2-k)} n^{2(k-1) /(2-k)} \leqq\left(M_{n_{0}}^{*}\right)^{2 k /(2-k)} \sum_{n=n_{0}} n^{2(k-1) /(2-k)} \\
& =K\left(M_{n_{0}}^{*}\right)^{2 k /(2-k)} n_{0}^{k /(2-k)}
\end{aligned}
$$

where $K$ is independent of $n_{0}$. But since $n_{0}$ is in $F_{y}$ we have

$$
\begin{aligned}
\left(\frac{y}{\|f\|_{l, \mu}}\right)^{l} & <\left(\left\{\left(M_{n_{0}}^{*}\right)^{1+k /(k-2)}\right\}\left\{n_{0}^{(k-1) /(k-2)}\right\}\right)^{l}=\left(M_{n_{0}}^{*}\right)^{2(k-1) l /(k-2)} n_{0}^{(k-1) l /(k-2)} \\
& =\left(M_{n_{0}}^{*}\right)^{2 k /(k-2)} n_{0}^{k /(k-2)} .
\end{aligned}
$$

Thus, taking reciprocals, and using the above inequality involving $\xi\left(F_{y}\right)$, we obtain (3.4) and, thus, the theorem is proved.

By considering adjoint transformations we can obtain, from this last theorem, another generalized Paley theorem. We proceed as follows:

If $(R, R, r)$ is a $\sigma$-finite measure space and $1 / p+1 / q=1, p \geqq 1$, the $\mathrm{F}$. Riesz representation theorem asserts that if we are given a bounded linear functional, $\mathscr{L}$, on $L^{p}$, there exists a $g$ in $L^{q}$ such that $\mathscr{L}(f)=\int_{R} f g d r$ for all $f$ in $L^{p}$ and, furthermore, $\|\mathscr{L}\|=\|g\|_{q, r}$. From now on we will restrict ourselves to $\sigma$-finite measure spaces (the following theorems are valid in more general settings but we shall not deal with such problems here). We put $\int_{R} f g d r$ $=\langle f, g\rangle$ for $f$ in $L^{p}$ and $g$ in $L^{q}$.

Let $\left\{\phi_{n}\right\}$ be the orthonormal system of Theorem (3.2) and $l<p \leqq 2$. Consider the linear transformation $A: L^{p}(M, \mathfrak{M}, \mu) \rightarrow l^{p}\left(l^{p}\right.$ is the class of all sequences $\left\{a_{n}\right\}$ with $\left.\sum\left|a_{n}\right|^{p}<\infty\right)$ that assigns to $f$ the sequence

$$
\left\{\left(M_{n}^{*}\right)^{k /(k-2)(1-2 / p)} n^{(k-1) /(k-2)(1-2 / p)} d_{n}\right\}
$$

where $d_{n}=\int_{M} f \phi_{n} d \mu$. Theorem (3.2) asserts that $A$ is bounded. Thus the adjoint, $A^{*}$ of $A$ must be a bounded linear transformation, with the same norm as $A$, defined on $l^{q}$ with range in $L^{q}(M, \mathfrak{M}, \mu)$. If $f$ is in $L^{p}(M, \mathfrak{M}, \mu)$ and $s=\left\{a_{n}\right\}$ is in $l^{q}$, we must have $\langle A f, s\rangle=\left\langle f, A^{*} s\right\rangle$, and, in particular, $\left\langle A \phi_{n}, s\right\rangle$ $=\left\langle\phi_{n}, A^{*} s\right\rangle$ for each $n$. But we see from the definition of $A$ that $\left\langle A \phi_{n}, s\right\rangle$ $=D_{n}^{(1-2 / p)} a_{n}$. On the other hand, since $A^{*}$ is bounded we must have

$$
\left\|A^{*}\right\|_{q, \mu} \leqq K\left(\sum_{n=1}^{\infty}\left|a_{n}\right|^{q}\right)^{1 / q} \text {. }
$$

Letting $c_{n}=\left\langle A \phi_{n}, s\right\rangle=D_{n}^{(1-2 / p)} a_{n}$, we see that (3.5) is equivalent to

$$
\left\|A^{*} s\right\|_{q, \mu} \leqq K\left(\sum_{n=1}^{\infty}\left|c_{n}\right|^{q} D_{n}^{(2 q / p-q)}\right)^{1 / q} .
$$

But $2 q / p-q=q-2$. We thus have the following theorem.

THEOREM (3.6). Let $2 \leqq q<k$ and suppose that the series 


$$
\sum_{n=1}^{\infty}\left|c_{n}\right|^{q} D_{n}^{(q-2)}<\infty
$$

then there exists a unique function, $f$, in $L^{q}(M, \mathfrak{M}, \mu)$ such that

$$
\int_{M} f \phi_{n} d \mu=c_{n}
$$

for all $n$, and furthermore,

$$
\|f\|_{q, \mu} \leqq K\left(\sum_{n=1}^{\infty}\left|c_{n}\right|^{q} D_{n}^{(q-2)}\right)^{1 / q} .
$$

The best constant, $K$, in (3.7) is the same as the best constant in (3.3) for the index $p$ satisfying $1 / p+1 / q=1$.

Now let us suppose that the measure space $(M, \mathfrak{M}, \mu)$ is an interval in the real line with Lebesgue measure. We shall assume for simplicity that the interval has the form $(0, \infty)$. It can easily be shown that the following theorems, with appropriate modifications, hold for any real interval.

We again suppose that $\left\{\phi_{n}\right\}$ is an orthonormal system satisfying (3.1). If we interchange in Theorems (3.2) and (3.6) the role of $f$ and the coefficients $c_{n}$ we obtain generalizations of two more Paley theorems that were also obtained in [5]. Theorem (3.9) below can be obtained from Theorem (2.9) in a manner completely analogous to the proof of Theorem (3.2). (3.8) is just the adjoint of (3.9).

TheOREM (3.8). Let $q \geqq 2$ and suppose that $f$ is in $L^{q}(0, \infty)$ with respect to the measure $d \mu=x^{(q-2) / l} d x$. Let $c_{n}=\int_{0}^{\infty} f \phi_{n} d x$ be the Fourier coefficients of $f$ with respect to $\left\{\phi_{n}\right\}$. Then

$$
\left(\sum_{n=1}^{\infty}\left|c_{n}\right|^{q} M_{n}^{2-q}\right)^{1 / q} \leqq K_{q, l}\left(\int_{0}^{\infty}|f(x)|^{q} x^{(q-2) / l} d x\right)^{1 / q} .
$$

Theorem (3.9). Suppose $1<p \leqq 2$ and that both $\sum\left|c_{n}\right|{ }^{p} M_{n}^{2-p}$ and $\sum\left|c_{n}\right|^{2}$ are finite. Then there exists a function $f$ with Fourier coefficients $c_{n}$ satisfying

$$
\left(\int_{0}^{\infty}|f(x)|^{p} x^{(p-2) / l} d x\right)^{1 / p} \leqq K_{p, l}\left(\sum_{n=1}^{\infty}\left|c_{n}\right|^{p} M_{n}^{2-p}\right)^{1 / p} .
$$

It is interesting to note that these two theorems do not involve the constants $M_{n}^{*}$ of Theorems (3.2) and (3.6). It is still an open question if these two latter theorems hold when the $D_{n}$ 's are defined with the constants $M_{n}$ in place of the $M_{n}^{*}$ 's.

4. A generalization of a theorem of $\mathrm{H}$. R. Pitt. From now on, if $p \geqq 1$, $p^{\prime}$ will denote the number satisfying $1 / p+1 / p^{\prime}=1$.

Let $f(x)$ be defined on $(-\pi, \pi)$ and suppose that $f(x)$ is in $L^{1}(-\pi, \pi)$; let 


$$
a_{n}=\int_{-\pi}^{\pi} f(x) e^{-i n x} d x
$$

H. R. Pitt has proved the following [6]:

Theorem (4.1).

$$
\left(\sum_{-\infty}^{\infty}\left|a_{n}\right|^{q}(|n|+1)^{-\lambda q}\right)^{1 / q} \leqq A\left(\int_{-\pi}^{\pi}|f(x)|^{p}|x|^{\alpha p} d x\right)^{1 / p}
$$

whenever $0 \leqq \alpha<1 / p^{\prime}, q \geqq p$ and $\lambda=1 / q+1 / p-1+\alpha \geqq 0$.

The following special cases are noteworthy:

(i) when $\alpha=\lambda=0$ then $q=p^{\prime}$ and we have the Hausdorff-Young inequality.

(ii) when $q=p$ and $\alpha=0$ then we have the inequality of Hardy and Littlewood; similarly for $q=p$ and $\lambda=0$.

One of us [8] has shown that (4.1) can be generalized to a theorem involving Fourier coefficients with respect to an arbitrary, uniformly bounded, orthonormal system defined on a real interval. It is our aim to extend this last theorem, and, hence, Pitt's theorem, to include orthonormal systems satisfying (3.1).

Let us, then, suppose that $\left\{\phi_{n}(x)\right\}$ is an orthonormal system satisfying (3.1) with respect to Lebesgue measure on the interval $(0,1)$. We then have the following

THEOREM (4.2). Let $f(x)$ be a function on $(0,1)$ with Fourier coefficients $c_{n}$ with respect to $\left\{\phi_{n}\right\}$. Then

$$
\left(\sum_{n=1}^{\infty}\left|c_{n}\right|^{q} D_{n}^{-\lambda q} M_{n}^{-\lambda q-(q-2)}\right)^{1 / q} \leqq A\left(\int_{0}^{1}|f(x)|^{p}|x|^{\alpha p / l} d x\right)^{1 / p}
$$

where $l<p, q \geqq p, 0 \leqq \alpha<1 / p^{\prime}-1 / k, \lambda=l / p+(2-l) / q-1+\alpha \geqq 0$ and $A$ depends on $l, \alpha, p$ and $q$.

We observe that if in (4.3) we put $p=q$ and $\lambda=0$ we obtain Theorem (3.8). For $p=q, \alpha=0$ (4.3) becomes (3.3). When $\alpha=\lambda=0$ and $l=1$ we obtain a generalization of the Hausdorff-Young theorem. The interval $(0,1)$ is not essential, any interval in the real line would do. If the interval is of the form $(0, a),(4.3)$ is unchanged provided $\int_{0}^{a}$ is substituted for $\int_{0}^{1}$. For other intervals the measure $x^{(q-2) / l} d x$ has to be slightly altered.

Proof of Theorem (4.2). We simplify the argument by breaking up the proof into several special cases, as was done in [8]. These special cases are the most interesting ones included in (4.3).

The proof will involve various applications of Theorems (2.9) or (2.11) (either of these will do). The chief "end points" results are (3.3) and (3.8), which we rewrite here for handy reference. 


$$
\left(\sum_{n=1}^{\infty}\left|c_{n}\right|^{p} D_{n}^{p-2}\right)^{1 / p} \leqq K_{p, k}\left(\int_{0}^{1}|f(x)|^{p} d x\right)^{1 / p}
$$

where $l<p \leqq 2$, and

$$
\left(\sum_{n=1}^{\infty}\left|c_{n}\right|^{q} M_{n}^{2-q}\right)^{1 / q} \leqq A_{q, k}\left(\int_{0}^{1}|f(x)|^{q} x^{(q-2) / l} d x\right)^{1 / q}
$$

where $q \geqq 2$.

Case 1. $p=q=2$.

Here, and in the following cases, we shall always apply Theorem (2.11) (or (2.9)) to the operator $T$ mapping $f$ into its Fourier coefficients $c_{n}$ when we are given two inequalities asserting that $T$ is of types $\left(p_{0}, q_{0}\right)$ and $\left(p_{1}, q_{1}\right)$ with possibly different measures. We shall refer to this application by saying that we interpolate between these two inequalities.

Let $l<p \leqq 2$ and $\alpha=(2-p) / 2 p$, then $0 \leqq \alpha<1 / 2-1 / k$. Also let $q \geqq 2$ and interpolate between (1) and (2) with $b_{0}(n)=D_{n}^{(p-2) / p}, b_{1}(n)=M_{n}^{(2-q) / q}, a_{0}(x)$ $=1$, and $a_{1}(x)=x^{(q-2) / q t}$. For $t=1 / 2$ we obtain

$$
\left(\sum_{n=1}\left|c_{n}\right|^{2} D_{n}^{-2 \alpha} M_{n}^{-2 \alpha}\right)^{1 / 2} \leqq A_{\alpha}\left(\int_{0}^{1}|f(x)|^{2} x^{2 \alpha / l} d x\right)^{1 / 2}
$$

for $0 \leqq \alpha<1 / 2-1 / k$ and $\lambda=\alpha$, which is (4.3) in the case $p=q=2$.

Case 2. $1<p=q \leqq 2$.

Rewrite (3) in the form

$$
\left(\sum_{n=1}^{\infty}\left|c_{n}\right|^{2} D_{n}^{-2 \beta} M_{n}^{-2 \beta}\right)^{1 / 2} \leqq A_{\beta}\left(\int_{0}^{1}|f(x)|^{2} x^{2 \beta / 2} d x\right)^{1 / 2}
$$

with $0 \leqq \beta<1 / 2-1 / k$.

Now suppose $p, \alpha, \lambda$ are given with $l<p \leqq 2, \alpha<1 / p^{\prime}-1 / k$ and $\lambda=2 / p-1$ $+\alpha \geqq 0$. Put in (1) the index $p_{0}$, where $1 / p_{0}=1 / p+\alpha$. Then we must have $1 / l>1 / p_{0} \geqq 1 / 2$. In ( $\left.3^{\prime}\right)$ let $\beta=1 / p+\alpha-1 / 2=1 / p_{0}-1 / 2$. We thus have $\beta<1 / l-1 / 2=1 / 2-1 / k$, since $1 / p_{0}<1 / l$, and, also, $\beta \geqq 0$, since $1 / p_{0} \geqq 1 / 2$. Thus the conditions on $\beta$ in $\left(3^{\prime}\right)$ are satisfied. Let $t=\alpha /(1 / p+\alpha-1 / 2)=\alpha / \beta$, then, clearly, $0 \leqq t \leqq 1$. Interpolating between (1) and $\left(3^{\prime}\right)$ we obtain:

$$
\left(\sum_{n=1}^{\infty}\left|c_{n}\right|^{p}\left\{D_{n}^{-\alpha} M_{n}^{-\alpha} D_{n}^{\left(p_{0}-2\right) / p_{0}(1-\alpha / \beta)}\right\}^{p}\right)^{1 / p} \leqq A_{p, \alpha}\left(\int_{0}^{1}|f(x)|^{p}\left(x^{\alpha / l}\right)^{p} d x\right)^{1 / p} .
$$

But $-\alpha p+p(1-\alpha / \beta)\left(p_{0}-2\right) / p_{0}=-\lambda p$ and $-\alpha p=-\lambda p+2-p$. Thus the last inequality becomes

$$
\left(\sum_{n=1}^{\infty}\left|c_{n}\right|^{p} D_{n}^{-\lambda p} M_{n}^{-\lambda p-(p-2)}\right)^{1 / p} \leqq A_{p, \alpha}\left(\int_{0}^{1}|f(x)|^{p} x^{\alpha p / l} d x\right)^{1 / p}
$$

for $l<p \leqq 2, \alpha<1 / p^{\prime}-1 / k$ and $\lambda=2 / p-1+\alpha \geqq 0$. 
Case 3. $l / p+(2-l) / q=1, l<p \leqq 2$.

Here we make use of

$$
\sup _{n}\left\{\left|c_{n}\right| / M_{n}\right\} \leqq\left(\int_{0}^{1}|f(x)|^{l} d x\right)^{1 / l} .
$$

If $p$ and $q$ are given and satisfy the above conditions, let $t$ be such that $1 / p=(1-t) / l+t / l$, then $0<t \leqq 1$, and let $\beta t=\alpha$. We now interpolate between $\left(3^{\prime}\right)$ and $(5)$ obtaining:

$$
\left(\sum_{n=1}^{\infty}\left|c_{n}\right|^{q} M_{n}^{-[\alpha+(1-t)] q} D_{n}^{-\alpha q}\right)^{1 / q} \leqq A_{p, \alpha}\left(\int_{0}^{1}|f(x)|^{p} x^{\alpha p / l} d x\right)^{1 / p}
$$

where $l / p+(2-l) / q=1, l<p \leqq 2$ and $0 \leqq \alpha<1 / l-1 / p$.

But, since $1 / q=t / 2,(1-t) q=q-2$. Thus we have:

$$
\left(\sum_{n=1}^{\infty}\left|c_{n}\right|^{q} M_{n}^{-\lambda q-(q-2)} D_{n}^{-\lambda q}\right)^{1 / q} \leqq A_{p, \alpha}\left(\int_{0}^{1}|f(x)|^{p} x^{\alpha p / l} d x\right)^{1 / p}
$$

where $l / p+(2-l) / q=1, l<p \leqq 2,0 \leqq \alpha<1 / p^{\prime}-1 / k$ and $\lambda=\alpha$.

Case $4 . l<p \leqq 2$.

Rewrite (4) as follows:

$$
\left(\sum_{n=1}^{\infty}\left|c_{n}\right|^{p} D_{n}^{-\mu p} M_{n}^{-\mu p+(2-p)}\right)^{1 / p} \leqq A_{p, \alpha}\left(\int_{0}^{1}|f(x)|^{p} x^{\alpha p / l} d x\right)^{1 / p}
$$

for $l<p \leqq 2,0 \leqq \alpha<1 / p^{\prime}-1 / k$ and $\mu=2 / p-1+\alpha \geqq 0$. Let $q_{t}=(1-t) / q+t / p$ for $0 \leqq t \leqq 1$ and interpolate between (6) and (7). We obtain:

$$
\begin{gathered}
\left(\sum_{n=1}^{\infty}\left|c_{n}\right|^{q_{t}}\left(M_{n}^{-(\lambda+1-2 / q)(1-t)} D_{n}^{-\alpha(1-t)} M_{n}^{-(\mu+1-2 / p) t} D_{n}^{-\mu t}\right)^{q_{t}}\right)^{1 / q_{t}} \\
\leqq A_{p, q_{t}, \alpha}\left(\int_{0}^{1}|f(x)|^{p} x^{\alpha p / l} d x\right)^{1 / p} .
\end{gathered}
$$

Let $\lambda=l / p+(2-l) / q_{t}-1+\alpha$. Then it can easily be checked, using the relationship $l / p+(2-l) / q=1$, that

$$
[(\alpha+1-2 / q)(1-t)+(\mu+1-2 / p) t] q_{t}=\lambda q_{t}+q_{t}-2
$$

and $\alpha(1-t) q_{t}+\mu t q_{t}=\lambda q_{t}$.

Thus we have:

$$
\left(\sum_{n=1}^{\infty}\left|c_{n}\right|^{q_{t}} D_{n}^{-\lambda q_{t}} M_{n}^{-\lambda q_{t}-\left(q_{t}-2\right)}\right)^{1 / q_{t}} \leqq A_{p, q_{t}, \alpha}\left(\int_{0}^{1}|f(x)|^{p} x^{p \alpha / l} d x\right)^{1 / p}
$$

for $l<p \leqq 2,0 \leqq \alpha<1 / p^{\prime}-1 / k, \lambda=l / p+(2-l) / q_{t}-1+\alpha \geqq 0$, and $q_{t} \geqq p$.

The last condition follows from the fact that since $l / p+(2-l) / q=1$ we have $q=((2-l) /(p-l)) p$, thus $q \geqq p$, and it follows that $q_{t} \geqq p$. 
Inequality (8) is (4.3) in case $l<p \leqq 2$. To obtain the case $p \geqq 2$ we can deduce a parallel sequence of inequalities. Instead of (4) we can obtain the analogous inequality for $p=q \geqq 2$ by interpolating between (2) and (3). We can then use this result and (5) to obtain the remainder of the theorem in a way similar to that yielding (8). This completes the proof of (4.2).

By taking the adjoint operators to the ones occurring in Theorem (4.2) we obtain another interesting theorem relating the Fourier coefficients and their associated functions. Such a theorem would contain Theorems (3.6) and (3.9) as special cases. We shall, however, let the matter stand here.

\section{REFERENCES}

1. Calderon and Zygmund, $A$ note on the interpolation of sublinear operators, Amer. J. Math. vol. 78 (1956) pp. 282-288. 56.

2. I. I. Hirschman, Jr., A note on orthogonal systems, Pacific J. Math. vol. 6 (1956) pp. 47-

3. Kolmogoroff, Sur les functions harmoniques conjuguées et les seriés de Fourier, Fund. Math. vol. 7 (1925).

4. Marcinkiewicz, Sur l'interpolation d'operations, C. R. Acad. Sci. Paris, vol. 208 (1939) pp. 1272-1273.

5. Marcinkiewicz and Zygmund, Some theorems on orthogonal systems, Fund. Math. vol. 28 (1937) pp. 309-335.

6. H. R. Pitt, Theorems on Fourier and power series, Duke Math. J. vol. 3 (1937) pp. 747755.

7. M. Riesz, Sur les maxima des formes bilinéares et sur les fonctionelles linéaires, Acta Math. vol. 29 (1926) pp. 465-497.

8. E. Stein, Interpolation of linear operators, Trans. Amer. Math. Soc. vol. 83 (1956) pp. $482-492$.

9. Thorin, Convexity theorems, Uppsala, 1948, pp. 1-57.

10. Zygmund, Trigonometrical series, Warsaw, 1935.

11. - On the Marcinkiewicz interpolation theorem, to appear in J. Math. Pures Appl.

University of Chicago,

Chicago, Ill.

De Paul University,

Chicago, Ill. 